\title{
A Quadrupole Mass Spectrometer for Resolution of Low Mass Isotopes
}

\author{
Jeyan Sreekumar, ${ }^{\text {Thomas J. Hogan, }}{ }^{\text {T Stephen Taylor, }}{ }^{\text {a }}$ Phillip Turner, \\ and Christopher Knott ${ }^{\mathrm{b}}$ \\ a Department of Electrical Engineering and Electronics, University of Liverpool, Liverpool, United Kingdom \\ ${ }^{b}$ AWE Aldermaston, Berkshire, United Kingdom
}

\begin{abstract}
The qualitative and quantitative identification of low mass isotopes in the mass range $1-6 \mathrm{u}$ poses certain difficulties when attempting to achieve the required resolution with an instrument suitable for deployment within a process environment. Certain adjacent species present in the process sample $\left(\mathrm{HT}\right.$ and $\left.\mathrm{D}_{2}\right)$ require a resolution greater than 930 to achieve an accurate measurement. We demonstrate here through simulation techniques that this level of performance required is unachievable using commercially available instruments. Using previously reported simulation techniques, this article demonstrates how the required performance for resolving the low mass isotopes can be achieved by a quadrupole mass spectrometer (QMS), which incorporates a quadrupole mass filter (QMF) constructed from hyperbolic electrodes and operated in zone 3 of the Mathieu stability diagram. (J Am Soc Mass Spectrom 2010, 21, 1364-1370) (c) 2010 Published by Elsevier Inc. on behalf of American Society for Mass Spectrometry
\end{abstract}

$\mathrm{M}$ ost commercial quadrupole mass spectrometers (QMS) utilize a quadrupole mass filter (QMF) based on circular rods and operate in stability zone 1 ( $a=0.237, q=0.706)$ [1]. A mass scan line that provides a mass resolution, which increases linearly with mass and commonly referred to as unit resolution, is the default mass scanning mode. There are a number of applications that require a high-resolution at the lower end of the mass range i.e., $1-6 \mathrm{u}$, where 1 atomic mass unit $(\mathrm{u})=1.6605 \times 10^{-27} \mathrm{~kg}$ [2]. Example applications include the quantitative measurement of hydrogen isotopes in the presence of helium [3-6] and helium leak detection in a deuterium rich atmosphere [7]. For instance, to measure ${ }^{3} \mathrm{He}^{+}$in the presence of $\mathrm{HD}^{+}$requires a minimum baseline resolution of 512 and for $\mathrm{HT}^{+}$in the presence of $\mathrm{D}_{2}{ }^{+}, 930$ is required. These resolutions are not readily achievable with commercial instruments suitable for deployment in a process environment. A list of the species and the resolutions to separate adjacent species is contained in Table 1.

General purpose instruments are also arranged to have relatively large mass ranges $100-300 \mathrm{u}$ being common. These characteristics result in relatively coarse control of the mass step size, e.g., 64 mass steps $/ u$ is common [8]. The ability to achieve highresolution quantitative measurements of closely spaced species requires a high number of data points or mass steps across the mass peaks. A small mass step size

Address reprint requests to Dr. S. Taylor, Department of Electrical Engineering and Electronics, University of Liverpool, Brownlow Hill, Liverpool L69 3GJ, United Kingdom. E-mail: s.taylor@liv.ac imposes a reduced maximum mass due to the finite dynamic range of digital to analogue (d/a) converters in the control electronics. This relationship has been demonstrated by Day [8], who used a modified QMS to successfully baseline separate ${ }^{4} \mathrm{He}^{+}$and $\mathrm{D}_{2}{ }^{+}$.

For a given length QMF the resolving power is dependent on the number of $\mathrm{rf}$ cycles the ion is exposed to on its passage through the QMF [9]. Typically $200 \mathrm{rf}$ cycles are required in zone 1 to achieve a resolution of $\approx 1000$, but this maximum is dependent on the overall accuracy of the QMF and the drive electronics, with any inaccuracies reducing this value [9]. For a QMF length of $300 \mathrm{~mm}$, a $2 \mathrm{u}$ ion with energy of $5 \mathrm{eV}$ would be exposed to 75 cycles, and a $6 \mathrm{u}$ ion to 120 cycles. Clearly, this is insufficient to achieve our required resolution described above, even with perfect electrode profiles, assembly, and voltage accuracies.

Stability zone 3 is a sloping rectangular area that provides two tips, one at the lower right $(a=2.52, q=$ $2.82)$, and the second at the upper left $(a=3.16, q=$ 3.23) corners that enable high-resolution mass scanning to be achieved.

With zone 3 the ions require exposure to a lower number of $\mathrm{rf}$ cycles to achieve the same resolution compared with zone 1 [9]. However, due to the higher Mathieu coefficients associated with zone 3, increased operating voltages are required. This, to some extent, is mitigated in this application by the limited maximum operating mass of $6 \mathrm{u}$ that is required for hydrogen isotope measurement. Due to the much narrower stability zone the sensitivity of a zone 3 operating instrument is reduced (compared with a zone 1 QMS), with a reduction of a factor of 50 for $\mathrm{He}^{+}$being quoted by 
Table 1. Minimum resolution required to discriminate between each of the species present

\begin{tabular}{|c|c|c|c|c|c|c|c|c|}
\hline \multirow[b]{2}{*}{ Species } & \multirow[b]{2}{*}{ Mass (Da) } & \multicolumn{7}{|c|}{ Minimum resolution } \\
\hline & & $\begin{array}{c}\mathrm{H}_{2}^{+} \\
2.01565\end{array}$ & $\begin{array}{c}{ }^{3} \mathrm{He}^{+} \\
3.01602932\end{array}$ & $\begin{array}{c}\mathrm{HD}^{+} \\
3.021926779\end{array}$ & $\begin{array}{c}{ }^{4} \mathrm{He}^{+} \\
4.002602\end{array}$ & $\begin{array}{c}\mathrm{HT}^{+} \\
4.02387427\end{array}$ & $\begin{array}{c}\mathrm{D}_{2}^{+} \\
4.0282036\end{array}$ & $\begin{array}{c}\mathrm{T}_{2}^{+} \\
6.03209854\end{array}$ \\
\hline $\mathrm{H}_{2}^{+}$ & 2.01565 & NA & 3.0 & 3.0 & 2.0 & 2.0 & 2.0 & 1.5 \\
\hline${ }^{3} \mathrm{He}^{+}$ & 3.016029319 & 2.0 & NA & 512.4 & 4.1 & 4.0 & 4.0 & 2.0 \\
\hline $\mathrm{HD}^{+}$ & 3.021926779 & 2.0 & 511.4 & NA & 4.1 & 4.0 & 4.0 & 2.0 \\
\hline${ }^{4} \mathrm{He}^{+}$ & 4.002602 & 1.0 & 3.1 & 3.1 & NA & 189.2 & 157.3 & 3.0 \\
\hline $\mathrm{HT}^{+}$ & 4.02387427 & 1.0 & 3.0 & 3.0 & 188.2 & NA & 930.5 & 3.0 \\
\hline $\mathrm{D}_{2}^{+}$ & 4.028203558 & 1.0 & 3.0 & 3.0 & 156.3 & 929.5 & NA & 3.0 \\
\hline $\mathrm{T}_{2}^{+}$ & 6.03209854 & 0.5 & 1.0 & 1.0 & 2.0 & 2.0 & 2.0 & NA \\
\hline
\end{tabular}

Hiroki [10]. Hiroki also reported that the upper corner of zone 3 was the most suitable for high-resolution operation [5] but that the lower corner provided increased sensitivity.

Circular rods provide a more cost-effective route to implementing a QMF but suffer from reduced performance when compared to hyperbolic rods. Computer simulation has shown that when operating in zone 1 , circular rods with $\mathrm{r} / \mathrm{r}_{0}=1.148$ suffer from increased peak width, lower transmission, and low mass tailing than hyperbolic rods [11]. More recent work has shown that this is also true when values of $\mathrm{r} / \mathrm{r}_{0}=1.125$ to 1.127 are used, which have been shown to give a better approximation to the quadrupole field than $\mathrm{r} / \mathrm{r}_{0}=1.148$ [12-14]. Using our simulation program QMS2, with 60 cycles of rf, a comparison of resolution at $10 \%$ peak height and transmission through QMS using filters are shown in Table 2. Performance degradation is further reduced when operating in zone 3 [15].

We have used our previously reported simulation techniques $[15,16]$ to investigate the factors that influence the ability of a QMS to achieve the performance necessary to separate $\mathrm{HT}^{+}$in the presence of $\mathrm{D}_{2}{ }^{+}$. Due to the demanding requirements of this application and for the reasons discussed above, the use of circular electrodes was felt to be inadequate for the performance required, and the results achieved in this study are based on a hyperbolic rod QMS. Our results show that frequency length product is a deciding factor in achieving the required resolution. It is also necessary for very accurate control of the mass scan line to ensure adequate abundance sensitivity. Further, we show that accurate alignment of the ion source to the QMF has to be achieved and this is more critical in the y-axis than in the $\mathrm{x}$-axis due to the narrower shape of the $\mathrm{y}$-stability area.

Table 2. Comparison of QMS resolution and transmission for values of $\mathrm{r} / \mathrm{r} 0$ and hyperbolic electrodes

\begin{tabular}{lcc}
\hline \multicolumn{1}{c}{$\mathrm{r} / \mathrm{r} 0$} & $\mathrm{R}(\mathrm{M} / \Delta \mathrm{M})$ & $\mathrm{T}(\%)$ \\
\hline \hline 1.148 & 56.91 & 9.1 \\
1.127 & 133 & 10.53 \\
Hyperbolic & 166.5 & 13.14 \\
\hline
\end{tabular}

\section{Theory}

A QMF with four equally spaced electrodes of hyperbolic form produces a central electric field whose strength increases linearly with increasing displacement from the central axis and is independent in $\mathrm{x}$ and $\mathrm{y}$ (ideal quadrupole field). When driven by the correct combination of dc and ac voltages, the electrode assembly is capable of providing mass filtering action. With the voltages defined in eqs 1 and 2, the behavior of the ions as they pass through the QMF can be defined by the Mathieu eq 3 [1].

$$
\begin{aligned}
& \Phi x=\frac{(U-V \cos \omega t)}{2} \\
& \Phi y=\frac{-(U-V \cos \omega t)}{2} \\
& \frac{d^{2} u}{d \xi^{2}}+\left(a_{u}-2 q u \cos (2 \xi)\right) u=0
\end{aligned}
$$

where $\mathrm{u}=\mathrm{x}$ or $\mathrm{y}$ and

$$
\begin{aligned}
& a_{u}=a_{x}=-a_{y}=\frac{4 e U}{m \omega^{2} r_{0}^{2}} \\
& q_{u}=q_{x}=-q_{y}=\frac{2 e U}{m \omega^{2} r_{0}^{2}}
\end{aligned}
$$

Where $m$ is the mass of the ion in $\mathrm{kg}$, e is electron charge in coulombs, $\omega$ is angular frequency of the $\mathrm{rf}$ voltage $\mathrm{V}$ in $\mathrm{MHz}, \mathrm{r}_{0}$ is radius of the central field aperture in $\mathrm{mm}$, $\mathrm{U}$ is dc component of the drive voltage in volts, and $\mathrm{V}$ is ac (rf) component of the drive voltage in volts.

Figure 1 shows the Mathieu stability diagram, which provides a graphical representation of the zones of the stable and unstable areas. This diagram shows the lowest three operating zones and for the purpose of this study we have examined zone $1(a=0.237, q=0.706)$ and zone $3(a=3.16, q=3.23)$.

The mass filtering action of the QMF is controlled by varying the $\mathrm{U}(\mathrm{dc})$ and $\mathrm{V}(\mathrm{rf})$ voltages. The voltage $\mathrm{V}$ 


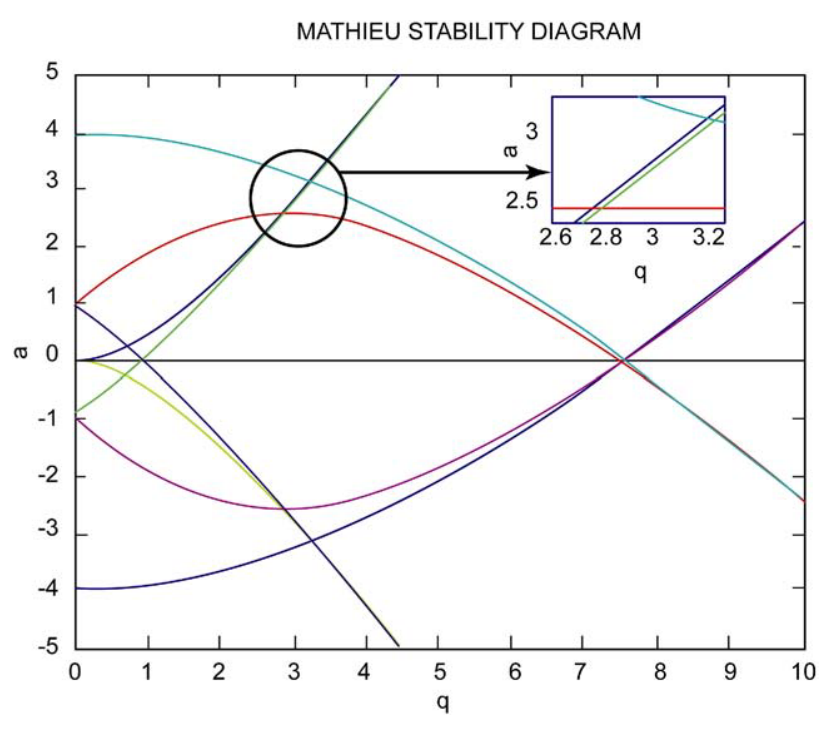

Figure 1. Mathieu stability diagram with zone 3 detail shown in the inset.

sets the position on the mass scale and the ratio of the $U$ and $\mathrm{V}$ voltages controls the instrument resolution setting $(\eta)$. Varying $\mathrm{V}$ and therefore $\mathrm{U}$ (while keeping the ratio of $\mathrm{U}$ to $\mathrm{V}$ constant) this changes the mass value that can successfully pass through the filter. This variation of the voltages is called the mass scan line. The ability of the QMF to provide the maximum achievable mass resolution is dependent on the number of $\mathrm{rf}$ cycles experienced by an ion. The number of rf cycles experienced by an ion can be defined by the following equation

$$
N_{c}=\frac{L f}{\sqrt{\frac{2 e V_{i}}{m}}}
$$

where $N_{c}$ is the number of rf cycles experienced by an ion of mass $m, L$ is length of the QMF in metres, $f$ is frequency of $\mathrm{rf}$ excitation in $\mathrm{MHz}, e$ electron charge in coulombs, $V_{\mathrm{i}}$ is accelerating voltage in volts, and $m$ is mass of ion in $\mathrm{kg}$.

\section{Method}

Custom software (QMS2-Hyperbolic) was used to simulate the performance of hyperbolic electrode QMF. The program calculates ion trajectories by solving the Mathieu equations using a fourth order Runge-Kutta algorithm. Our approach has been described previously $[15,16]$. Generation, display, and storage of mass spectra and individual ion trajectories, together with parametric sweep control, are provided by the QMS2Hyperbolic program. A second program, QMS2-ION generates the entry conditions for a large number of ions $\left(>10^{7}\right)$, which are stored in an ion file, and these ions are transmitted along mass filter axis, i.e., ion entrance angle is zero degrees. The number of ions $\left(10^{7}\right)$ generated is simulated at each mass scan step. This file is read by QMS2-Hyperbolic providing a uniform ion distribution across the ion source radius $R_{\mathrm{s}}$ with each ion having random phase with respect to the rf at the time of entry into the QMF. Finally, MatLab was used to post process the data and for the generation of the graphical results.

\section{Results and Discussion} Mass Spectral Studies for a $\mathrm{HT}^{+}$and $\mathrm{D}_{2}{ }^{+}$
Mixture

Reported here are the simulated mass spectra for a $\mathrm{HT}^{+}$ and $\mathrm{D}_{2}^{+}$mixture using the computer simulation software described and operated at the upper left hand tip of stability zone $3(\mathrm{a}=3.16$ and $\mathrm{q}=3.23)$. For zone 3 , amplitude and length of high mass tail reduces as the frequency increases with corresponding improvement in abundance sensitivity. Increasing frequency also reduces low mass tail but the effect is minimal. As previously noted, hyperbolic electrodes provide superior performance in resolution and ion transmission for a given number of $\mathrm{rf}$ cycles than circular or other shaped electrodes e.g., rectangular [17, 18]. The simulated performance characteristics of a QMF constructed from hyperbolic electrodes are therefore investigated. Our tests indicate that only stability zone 3 is capable of being able to achieve the desired performance in the mass range in question (1-6 u). The mass difference between the two species is $0.004329288 \mathrm{u}$, requiring a minimum resolution of 930 for mass discrimination. Increasing the frequency improves the resolution measured at $10 \%$ of spectrum peak height $(\mathrm{PH})$ and base line resolution but the $50 \% \mathrm{PH}$ resolution only increases for a limited range of frequencies before limiting. Due to the closeness of the two species, a higher resolution is required to separate $\mathrm{HT}^{+}$and $\mathrm{D}_{2}^{+}$ions. A higher resolution can be achieved only in the upper half of the operational mass range. This is due to the number of $\mathrm{rf}$ cycles that the two species experience being very similar. As the ion mass increases the velocity through the QMF falls (for a given ion energy), this gives an increase in the number of rf cycles experienced and an increase in achievable resolution.

Figure 2a shows mass spectra for $\mathrm{HT}^{+}$, at $10 \% \mathrm{PH}$ showing a resolution of 914 at $4 \mathrm{MHz}$, increasing to 1257 at $8 \mathrm{MHz}$. The corresponding 50\% $\mathrm{PH}$ resolution ranges from 1749 at the low-frequency end up to 1829 at $5 \mathrm{MHz}$ and above. Figure $2 \mathrm{~b}$ shows mass spectra for $\mathrm{D}_{2}{ }^{+}$showing a slightly higher resolution: the $10 \% \mathrm{PH}$ resolution ranges from 915 at the lowest frequency, up to a maximum of 1299 at $8 \mathrm{MHz}$. The $50 \% \mathrm{PH}$ resolution was also correspondingly greater, with a minimum 1751 at $4 \mathrm{MHz}$ increasing to 1918 at $6 \mathrm{MHz}$ and above. As was the case for $\mathrm{HD}^{+}$the $50 \% \mathrm{PH}$ resolution limits, with the higher mass $\mathrm{D}_{2}{ }^{+}$limiting at a slightly higher value than for the lower mass $\mathrm{HD}^{+}$. Both the low and 
high mass tails for the two species are low, but with the high mass tail having much greater amplitude and mass range. A minimum resolution of 930 is required to separate these two species $\mathrm{HT}^{+}$and $\mathrm{D}_{2}{ }^{+}$. This is achievable with a length of $300 \mathrm{~mm}$ operating at a frequency of $5 \mathrm{MHz}$. By using the previous $\mathrm{L} \times \mathrm{f}$ relationship (eq 6 ), this should also be achievable with a length of 250 $\mathrm{mm}$ operating at $6 \mathrm{MHz}$. Figure 3 shows the mass spectra for equal abundances of $\mathrm{HT}^{+}$and $\mathrm{D}_{2}{ }^{+}$. As previously discussed, the high mass tail is the more dominant. Therefore the high mass tail of the $\mathrm{HT}^{+}$mass peak is the most likely to be the cause of inter species interference. The valley between the two peaks decreases with increasing frequency. At the lower frequency, the minimum point on the valley represents $\sim 10 \%$ of peak height. For the highest frequency, this minimum drops to virtually zero.

Figure 4 shows typical mass spectra for $\mathrm{D}_{2}{ }^{+}$for operation on zone 1 using the computer simulation software described. The mass peaks show both low and
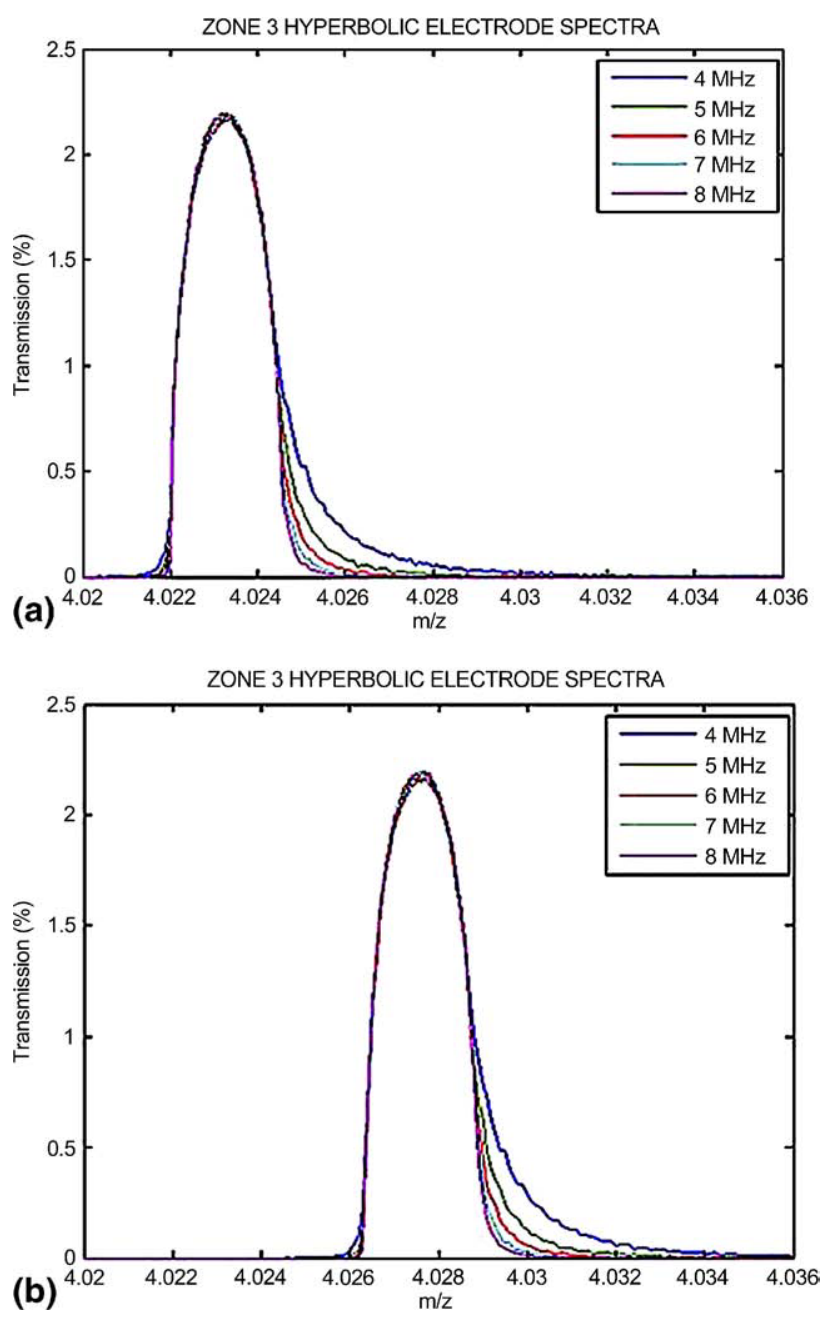

Figure 2. Variation of $\mathrm{HT}^{+}$mass spectra with frequency for a hyperbolic electrode QMF $\left(\mathrm{L}=300 \mathrm{~mm}, \mathrm{r}_{0}=2.76 \mathrm{~mm}, \mathrm{Ei}=15 \mathrm{eV}\right.$, ion source radius $=0.276 \mathrm{~mm})$ (a) and Variation of $\mathrm{D}_{2}{ }^{+}$mass spectra with frequency for a hyperbolic electrode QMF $(\mathrm{L}=300$ $\mathrm{mm}, \mathrm{r}_{0}=2.76 \mathrm{~mm}, \mathrm{Ei}=15 \mathrm{eV}$, ion source radius $\left.=0.276 \mathrm{~mm}\right)(\mathbf{b})$.

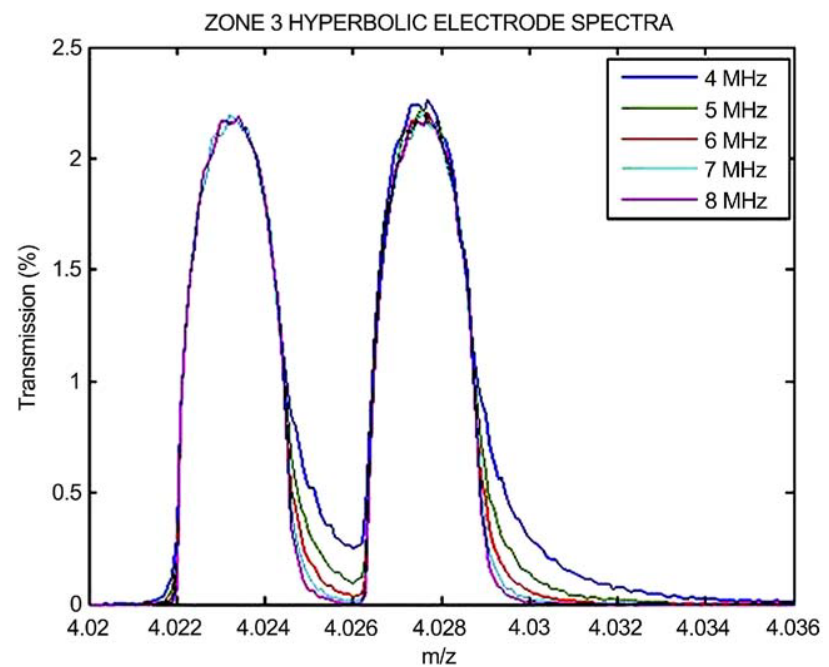

Figure 3. Variation of mass spectra with frequency for a hyperbolic electrode QMF ( $\mathrm{L}=300 \mathrm{~mm}, \mathrm{r}_{0}=2.76 \mathrm{~mm}, \mathrm{Ei}=15 \mathrm{eV}$, ion source radius $=0.276 \mathrm{~mm}$ ) for $\mathrm{HT}^{+}$and $\mathrm{D}_{2}{ }^{+}$ions with equal abundance.

high mass tails, which are well formed. In this case (zone 1) the low mass tail is of greater amplitude and width than the high mass tail. The $10 \% \mathrm{PH}$ resolution is significantly lower than obtained with zone 3 simulations. The resolution at the low-frequency end is 496 , compared with 915 for zone 3. As the frequency is increased, the difference in resolution between the two zones decreases. For the two higher frequencies in this mass range, zone 1 achieves a maximum of 1751 at 8 $\mathrm{MHz}$.

\section{Effects of Drive Voltage Tolerance}

Increasing the $\mathrm{U}$ and $\mathrm{V}$ drive voltages while keeping the ratio between them constant produces a constant reso-

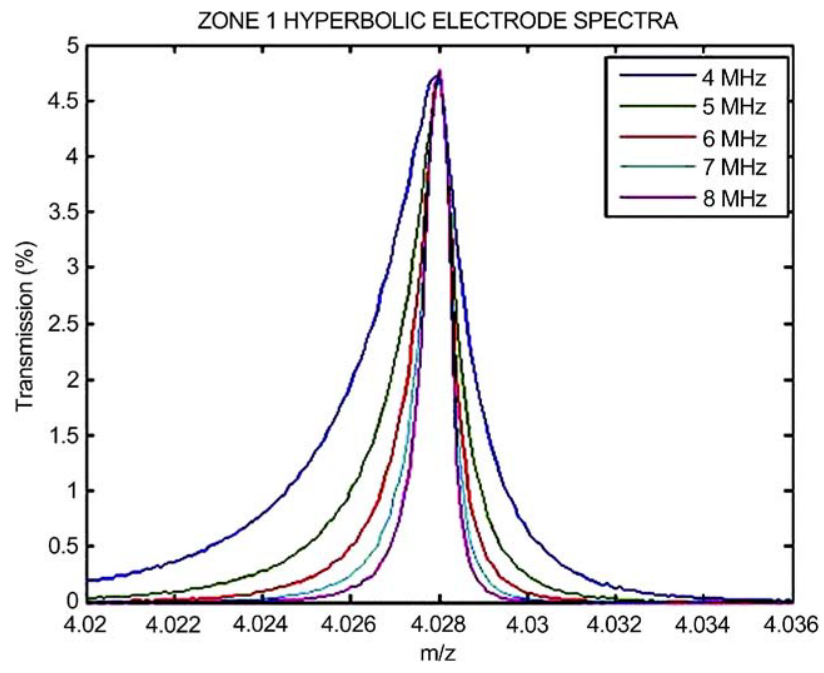

Figure 4. Variation of $\mathrm{D}_{2}{ }^{+}$zone 1 mass spectra $(\mathrm{L}=300 \mathrm{~mm}$, $\mathrm{r}_{0}=2.76 \mathrm{~mm}, \mathrm{Ei}=3 \mathrm{eV}$, ion source radius $0.276 \mathrm{~mm}$ for a range of $\mathrm{rf}$ frequencies. 
lution mass scan line (resolution proportional to mass). To achieve unit resolution the ratio of $U / V$ is varied with increasing mass and results in a more complex scan generation system. A shift in the mass scan line is observed as the ratio between the $U$ (dc) and V (rf) voltages varying which in turn results in a change to resolution and/or the mass calibration of the system. A very high instrument resolution $\eta$ is achieved with the tight control of the drive voltages. Changes to either $U$ or $\mathrm{V}$ independently results in a change to $\eta$ and small changes to peak shape results with minor changes to the inter peak valley shape. These small peak shape changes can occur due to small changes in the $U$ voltage. A greater change in instrument resolution $\eta$ produced as the tolerance limits increases the error in the ratio of $\mathrm{U}$ and $\mathrm{V}$ voltages and therefore increasing movement in the position of the scan line relative to the mass stability regions. This increased change in mass scan line position results in increasing distortion of the mass peak. As this voltage error increases the individual peaks widen with an accompanying increase in peak height. The inter peak valley amplitude increases finally disappearing with the two peaks merging.

Figure $5 \mathrm{a}$ and $\mathrm{b}$ show simulated mass spectra for $\mathrm{a}$ $\mathrm{HT}^{+}$and $\mathrm{D}_{2}{ }^{+}$mixture (50:50 ratio) for a range of negative $U$ drive voltage errors and the inset in Figure $5 \mathrm{a}$ and $\mathrm{b}$ show a correct scan line condition at $0.0 \mathrm{~V}$ and an incorrect scan line condition at $-0.1 \mathrm{~V}$. Normally a reduction in DC drive voltage (negative voltage error) due to the power supply tolerances, results in an increase in ion transmission and a broadening of the mass peak resulting in a reduction in resolution. At the lower end of the voltage error range considered (up to $-0.025 \mathrm{~V}$ ) the observable changes in the mass peak are minimal and acceptable. Above this value the valley between the two species rises resulting in discrimination at $10 \% \mathrm{PH}$ becoming unachievable and at a voltage error of $0.1 \mathrm{~V}$ the stability zones for the two species merge with the resultant superimposed peak emerging. At the negative voltage error of $-0.1 \mathrm{~V}$, the resolution has been reduced to a point where there is significant overlap between the stable zones of $\mathrm{HT}^{+}$and $\mathrm{D}_{2}{ }^{+}$ species as shown in the Figure $5 \mathrm{~b}$ inset. This results in poor mass discrimination. For this position of the mass scan line both low and high mass species are transmitted resulting in higher transmission accompanied by the appearance of the central peak. The drive voltage tolerance limit is clearly at its most stringent at this part of the mass scan range due to high-resolution required to discriminate between $\mathrm{HT}^{+}$and $\mathrm{D}_{2}{ }^{+}$. To maintain this performance it is necessary to control the $\mathrm{U} / \mathrm{V}$ ratio to very tight limits. The tolerance limits depend on the achievable mass resolution limit that can be obtained from the instrument.

\section{Ion Source Positional Displacements}

Previous research has characterized the dependence of QMF performance on electrode positional tolerance
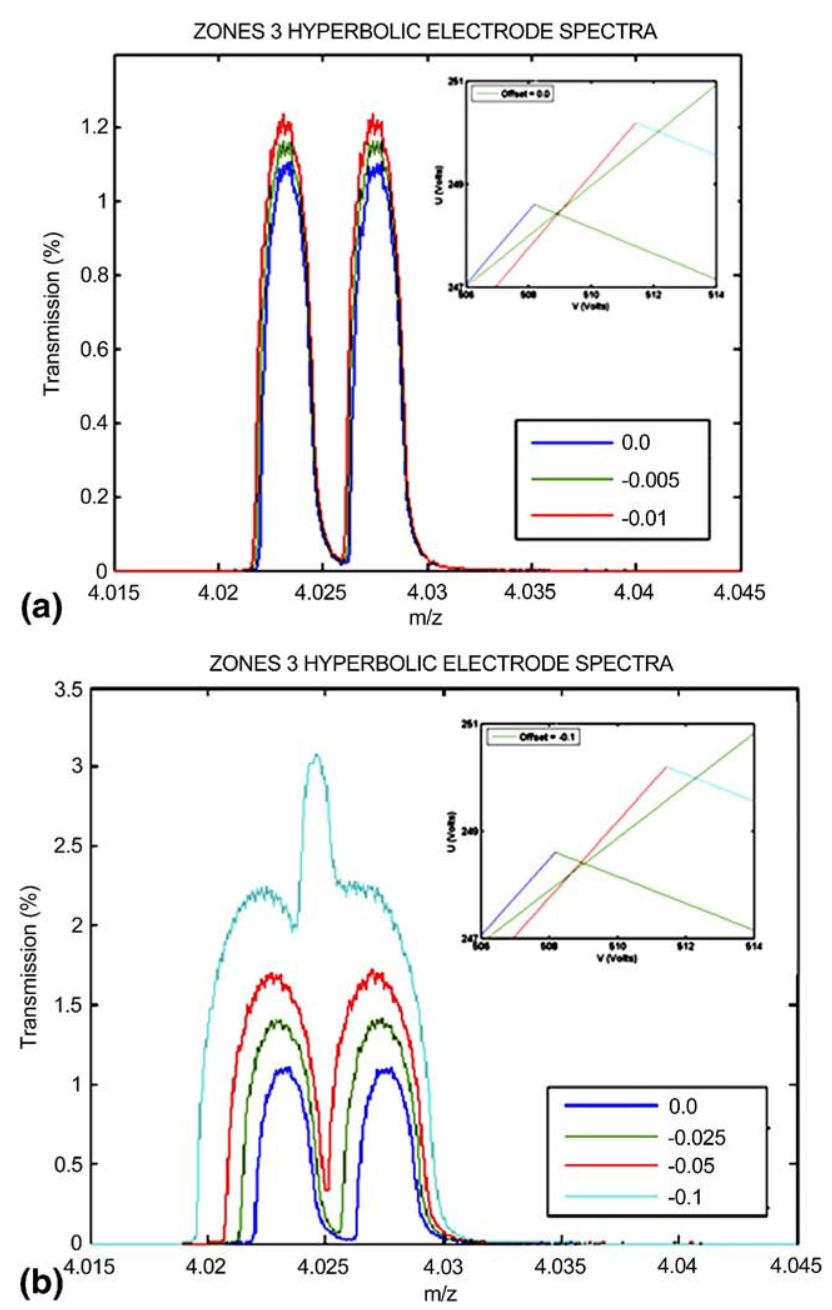

Figure 5. Mass spectra for a range of $U$ voltage acceptable tolerances (volts) (a) acceptable tolerances (b) unacceptable tolerances. Inset shows scan line for $\left({ }^{4} \mathrm{He} 4.0026 \mathrm{u}\right.$ and $\mathrm{D}_{2}$ 4.0282). Overlap of zones gives large central peak.

[19]. Here we show the degradation in performance that occurs for a range of QMF to ion source misalignments. Correct alignment of the ion source with the central axis of the QMF is important to ensure maximum sensitivity. Misalignments of $<0.2 \mathrm{~mm}$ (for an $\mathrm{r}_{0}$ of $2.76 \mathrm{~mm}$ ) results in a small reduction in the peak height (sensitivity) with barely discernible changes of the peak shape. For increasing misalignments the peak heights start to decrease significantly, decreasing by a factor of $>2$ for a displacement of $0.4 \mathrm{~mm}$ associated with an increasing narrowing of the peak width. Similar effects are observed for both $\mathrm{x}$ and $\mathrm{y}$ axis displacements. The $\mathrm{y}$ axis displacement results in greater decrease in peak height reflecting the narrower $y$-axis stable region of the QMF. We have also demonstrated that the effects of simultaneous $x$ and $y$ displacements are cumulative.

Figure $6 a$ and $b$ show mass spectra for a range of $x$ and $y$-axis displacement of the ion source with respect to the QMF. Both $x$ and y displacements exhibit similar characteristics with the y-axis displacement having the greater decrease in transmission for a 

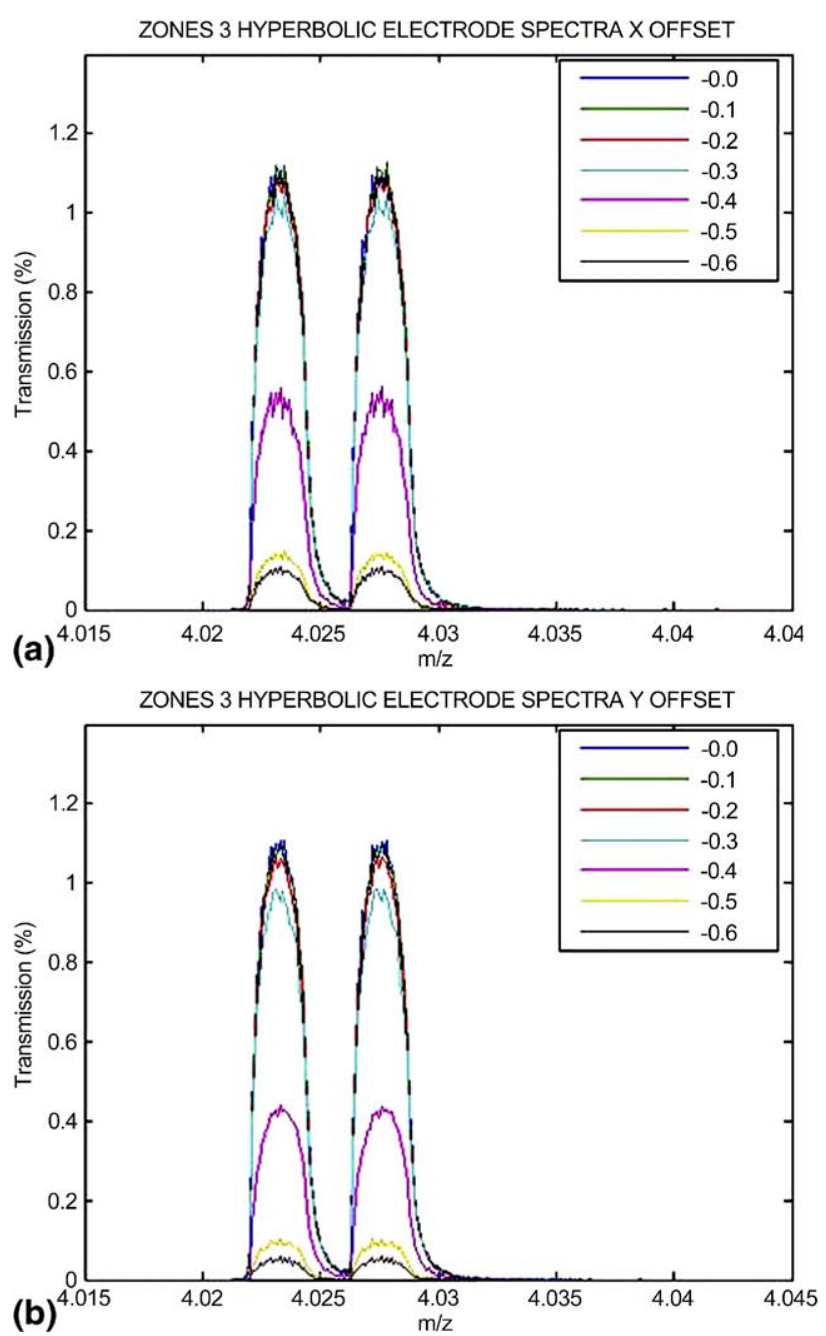

Figure 6. Mass spectra for a range of QMF to ion source alignment tolerances $(\mathrm{mm})\left(\mathrm{L}=300 \mathrm{~mm}, \mathrm{~F}=6 \mathrm{MHz}, \mathrm{r}_{0}=2.76\right.$ $\mathrm{mm}, \mathrm{Rs}=0.4 \mathrm{~mm}, \mathrm{Ei}=15 \mathrm{eV}$ ) upper $\mathrm{X}$ axis misalignment (a) and lower $\mathrm{Y}$ axis misalignment (b).

given displacement. Displacement in the positive direction results in a similar effect has confirmed this behavior and is due to the asymmetry of the QMF acceptance. For the condition tested, a tolerance of $\pm 0.2 \mathrm{~mm}$ would probably be acceptable. The effect of the fringing fields is not reported in the paper. This is the subject of continuing work requiring three dimensional modeling of the ion injection and extraction process from the QMF.

\section{Conclusions}

To provide adequate mass discrimination to resolve the peaks of hydrogen and helium isotopes presents certain difficulties. Due to their low mass, these isotopes have a relatively high velocity, which results in them experiencing a low number of $\mathrm{rf}$ cycles in their passage through the QMF and results in poor mass resolution with the average QMS. The use of hyperbolic electrodes is the best option to maximize the theoretical resolution, which is necessary for this application.
Our simulations show that the use of hyperbolic electrodes in conjunction with operation in stability zone 3 provides resolutions $>1000$, which exceeds the minimum required performance criteria for separation of $\mathrm{HT}^{+}$and $\mathrm{D}_{2}{ }^{+}$given in the introduction. The closeness of the species discussed demands high-resolution to achieve mass discrimination and to achieve adequate abundance sensitivity. This places tight constraints on the tolerance budget for the control electronics electrode drive system. The high mass tail has a greater effect than the lower mass tail on abundance sensitivity for these two species. Tighter control of the QMF to ion source alignment accuracy is required in the y-axis compared to the $\mathrm{x}$-axis due to the narrower $\mathrm{y}$-axis stability area. The results show that there is scope to optimize the choice of QMF length and drive frequency. We conclude that further work to include the design and manufacture of a prototype instrument is justified on the basis of the results reported here, and is necessary to investigate and quantify the performance characteristics experimentally.

\section{Acknowledgments}

The authors thank AWE Aldermaston UK for their support by sponsoring this project and to Neil France and Boris Brkić for undertaking experimental work that formed part of the overall project.

\section{References}

1. Dawson, P. H. The Mass Filter: Design and Performance. In Quadrupole Mass Spectrometry and Its Applications, Austin, W. E., Holme, A. E., Leck, J. H., Eds.; Elsevier: Amsterdam 1976; pp 121-152.

2. Kay, G. W. C.; Laby, T. H. Fundamental Constants. In Tables of Physical and Chemical constants and some Mathematical Functions, Longman: London and New York, 1986; pp 14-16.

3. Ellefson, R. E.; Moddeman, W. E.; Dylia, H. F. Hydrogen Isotope Analysis by Quadrupole Mass Spectrometry. J. Vac. Sci. Technol. 1981, 18, 1062-1066.

4. Hiroki, S.; Abe, T.; Murakami, Y. Separation of Helium and Deuterium Peaks with a Quadrupole Mass Spectrometer by Using the Second Stability Zone in the Mathieu Diagram. Rev. Sci. Instrum. 1992, 63(8), 83874-3876.

5. Hiroki, S.; Abe, T.; Murakami, Y. Detection of a $10^{-4}$ Helium Peak in a Deuterium Atmosphere Using a Modified High-Resolution Quadrupole Mass Spectrometer. Rev. Sci. Instrum. 1994, 65(6), 1912-1917.

6. Frattolillo, A.; De Ninno, A. A Powerful Tool to Quantitatively Detect Tiny Amounts of ${ }^{4} \mathrm{He}$ in a Deuterium Rich Background for Fusion Research. Proceedings of the 22nd IEEE Symposium on Fusion Engineering; Albuquerque, NM, June, 2007.

7. Hiroki, S.; Abe, T.; Murakami, Y. Sensitive Helium Leak Detection in a Deuterium Atmosphere Using a High-Resolution Quadrupole Mass Spectrometer. Vacuum 1996, 47(6/8), 767-769.

8. Day, C. The Use of a High-Resolution Quadrupole Mass Spectrometer System for Selective Detection of Helium and Deuterium. Vacuum 1998, 51(1), 21-30.

9. Du, Z.; Douglas, D. J.; Konenkov N. Elemental Analysis with Quadrupole Mass Filters Operated in Higher Stability Regions. J. Anal. At. Spectrom. 1999, 14(8), 1111-1119.

10. Hiroki, S.; Abe, T.; Murakami, Y.; Yanagishita, K.; Nakamura, S Development of a Quadrupole Mass Spectrometer Using the Second Stable Zone in Mathieu's Stability Diagram. Rev. Sci. Instrum. 1991, 62(9), 2121-2124.

11. Gibson, J. R.; Taylor, S. Prediction of Quadrupole Mass Filter Performance for Hyperbolic and Circular Cross Section Electrodes. Rapid Commun. Mass Spectrom. 2000, 14, 1669-1673.

12. Bracco, G. Comparison of Quadrupole Mass Filters Equipped with Rods of Different Convexity: An Analysis by Finite Element 
Methods and Trajectory Simulations. Int. J. Mass Spectrom. 2008, 278,75 .

13. Douglas, D. J.; Konenkov, N. V. Influence of the 6th and 10th Spatial Harmonics on the Peak Shape of a Quadrupole Mass Filter with Round Rods. Rapid Commun. Mass Spectrom. 2002, 16, 1425-1431.

14. Gibson, J. R.; Taylor, S. Numerical Investigation of the Effect of Electrode Size on the Behavior of Quadrupole Mass Filters. Rapid Commun. Mass Spectrom. 2001, 15, 1960-1964.

15. Hogan, T. J.; Taylor, S. Performance Simulation of a Quadrupole Mass Filter Operating in the First and Third Stability Zones. IEEE Trans. Instrum. Meas. 2008, 57(3), 498-508.
16. Hogan, T. J.; Taylor, S. Effects of Mechanical Tolerances on QMF Performance for Operation in the Third Stability Zone. IEEE Trans. Instrum. Meas. 2009, 99, doi:10.1109/TIM.2009.2028221.

17. Pearce, C. G.; Halsall, D. A Quadrupole Mass Filter with Flat Electrodes. Int. J. Mass Spectrom. Ion Phys. 1978, 27(1), 31-41.

18. Gibson, J. R.; Evans, K. G.; Taylor, S. Modeling Mass Analyzer Performance with Fields Determined Using the Boundary Element Method. J. Mass Spectrom. 2010, 45(4), 364-371.

19. Taylor, S.; Gibson, J. R. Prediction of the Effects of the Imperfect Construction of a QMS Filter. J. Mass Spectrom. 2008, 43(5), 609616. 